\title{
A NOTE ON PERIODIC SOLUTIONS OF FUNCTIONAL DIFFERENTIAL EQUATIONS
}

\author{
S. H. Chang \\ Department of Mathematics \\ Cleveland State University \\ Cleveland, Ohio 44115 \\ (Received September 15, 1983)
}

ABSTRACT. The existence of periodic solution for a certain functional differential equation with quasibounded nonlinearity is established.

KEY WORDS AND PHRASES. Quasibounded nonlinearity, periodic solution. 1980 MATHEMATICS SUBJECT CLASSIFICATION CODE. $34 \mathrm{~K} \mathbf{} 5$.

1. INTRODUCTION.

Let $C_{r}$ denote the Banach space of continuous $R^{n}$ - valued functions on $[-r, 0]$ with the supremum norm, i.e., for each $\phi \in C_{r},\|\phi\|=\max _{-r \leq \theta \leq 0}|\phi(\theta)|$. Also for a given continuous $R^{n}$ - valued function $x$ defined on $[-r, b)$ with $b>0$ and for $0 \leq t<b$, let $x_{t}$ be the function in $C_{r}$ defined by $x_{t}(\theta)=x(t+\theta)$ for all $\theta \in[-\mathrm{r}, 0]$.

Consider the following functional differential equation

$$
x^{\prime}(t)=L\left(t, x_{t}\right)+f\left(t, x_{t}\right)
$$

where $L$ and $f$ are continuous mappings from $[0, \infty) \times C_{r}$ into $R^{n}, L(t+T, \phi)=L(t, \phi)$ and $f(t+T, \phi)=f(t, \phi)$ for all $(t, \phi) \in[0, \infty) \times C_{r}$ and for some $T>0, L(t, \phi)$ is linear in $\phi$ for fixed $t$, and $f$ maps closed and bounded sets into bounded sets. Assume that the equation

$$
x^{\prime}(t)=L\left(t, x_{t}\right)
$$

has no nontrivial T-periodic solutions. Also, without loss of generality we assume $\mathrm{T} \geq \mathrm{r}$.

Fennel1 [2] has established the existence of $T$-periodic solution for the equation (1.1) by assuming

$$
\lim _{\|\phi\| \rightarrow \infty} \frac{|\mathrm{f}(\mathrm{t}, \phi)|}{\|\phi\|}=0
$$

uniformly in $t$. It is the purpose of this note to generalize Fennell's result by relaxing this requirement. We shall see that the limit in (1.3) can be allowed to be positive.

Using the mapping $f$ in (1.1), we give the following definition. The function 
$f$ is said to be quasibounded with respect to $\phi$ if the number

$$
|f|=\min _{0<\rho<\infty}\left(\max _{\substack{0 \leq t \leq T \\ \|}} \frac{|f(t, \phi)|}{\|\phi\|}\right)
$$

is finite; in this case, $|f|$ is called the quasinorm of $f$. In recent years, equations with quasibounded nonlinearities have been studied extensively. We shall show that if $\mathrm{f}$ is quasibounded and has a quasinorm smaller than a certain positive number then Eq. (1.1) has at least one T-periodic solution. Our proof uses a technique generalizing that used in [2].

2. THE RESULTS.

Under the assumption for (1.2), the functional differential equation

$$
x^{\prime}(t)=L\left(t, x_{t}\right)+h(t),
$$

where $L$ is the same as in (1.1) and $h:[0, \infty), R^{n}$ is continuous and T-periodic, has a unique T-periodic solution. Let $x(\psi, h): r-r, \infty) \rightarrow R^{n}$ denote the solution of (2.1) with initial value $\psi \cdot C_{r}$. Let $U: C_{r} \rightarrow C_{r}$ be the operator defined by $\mathrm{U} \phi=\mathrm{x}_{\mathrm{T}}(\phi, 0)$. Then $\mathrm{U}$ is completely continuous and the T-periodic solution of (2.1) is determined by the initial function $\psi=(I-U)^{-1} x_{T}(0, h)$. Let $\ell(t)$ be the norm of the operator $\mathrm{L}(t, \phi)$,

$$
E=\exp \left(\int_{0}^{T} \ell(s) d s\right)
$$

and

$$
\mathrm{K}=\mathrm{TE}^{2}\left\|(\mathrm{I}-\mathrm{U})^{-1}\right\|+\mathrm{TE} \text {. }
$$

THEOREM. If, in addition to the given assumptions for the equation $(1.1)$, $f$ is quasibounded with respect to $\phi$ and has a quasinorm $|\mathrm{f}|<1 / K$, where $K$ is given by (2.2), then (1.1) has at least one T-periodic solution.

PROOF. The following inequality

$$
\left\|x_{t}(\phi, h)\right\| \leq\left\{\|\phi\|+\int_{0}^{t}|h(s)| d s\right\} \exp \left(\int_{0}^{t} \ell(s) d s\right), t \geq 0,
$$

which follows from (2.1) and Gronwall's lemma, will be needed.

Let $X$ be the Banach space of continuous $T$-periodic functions from $[-r, \infty)$ into $R^{n}$ with the supremum norm. For each $\phi \in X$, let $\hat{f}(\phi)(t)=f\left(t, \phi_{t}\right)$. Then $\hat{\mathrm{f}}(\phi):[0, \infty) \rightarrow \mathrm{R}^{\mathrm{n}}$ is continuous and T-periodic. Let $\psi=(I-U)^{-1} \mathrm{x}_{T}(0, \hat{\mathrm{f}}(\phi))$. Then $\psi \in C_{r^{*}}$ Now, define a mapping $P: X \rightarrow X$ by $P \phi=x(\psi, \hat{f}(\phi))$, i.e., $P \phi$ is the unique T-periodic solution of

Then $P$ is a continuous mapping.

$$
x^{\prime}(t)=L\left(t, x_{t}\right)+f\left(t, \phi_{t}\right) .
$$

Since $|\mathrm{f}|<1 / \mathrm{K}$, there exists $\varepsilon>0$ such that $|\mathrm{f}|+\varepsilon<1 / \mathrm{K}$. Then by the definition of quasiboundedness (1.4) there exists $\rho(\varepsilon)>0$ such that

$$
\frac{|f(t, \phi)|}{\|\phi\| !}<\frac{1}{K} \text { whenever }\|\phi\| \geq \rho(\varepsilon) \text { and } 0 \leq t \leq T \text {. }
$$


Let

$$
N=\max \left\{|f(t, \phi)|: \phi \in C_{r},\|\phi\| \leq \rho(\varepsilon), 0 \leq t \leq T\right\} .
$$

Then let $M=\max \{K N, \rho(\varepsilon)\}$ and

$$
D=\{\phi \in X:\|\phi\| \leq M\} .
$$

We claim that (i) $P(D) \subset D$ and (ii) $P(D)$ is relatively compact.

Using the inequality (2.3), we obtain that

$$
\|P \phi\|=\max _{0 \leq t \leq T}|P \phi(t)| \leq K \max _{0 \leq s \leq T}\left|f\left(s, \phi_{s}\right)\right| \text {. }
$$

Now for $\phi \in D$ and $0 \leq s \leq T$, if $\left\|\phi_{S}\right\| \leq \rho(\varepsilon)$ then $K\left|f\left(s, \phi_{s}\right)\right| \leq K N \leq M$ and if $\left\|\phi_{s}\right\|>\rho(\varepsilon)$ then $\mathrm{K}\left|\mathrm{f}\left(\mathrm{s}, \phi_{\mathrm{s}}\right)\right|<\left\|\phi_{\mathrm{s}}\right\| \leq\|\phi\| \leq M$. Thus $\|\mathrm{P} \phi\| \leq M$ whenever $\phi \epsilon \mathrm{D}$. This proves (i). (ii) can be established by using an argument similar to that used in [2].

By Schauder's fixed point theorem ([3], or see [1, p. 131]) there exists $\phi \in D$ such that $P \phi=\phi$, which completes the proof of the theorem.

COROLLARY (FENNELL [2]). If, in addition to the given assumptions for the equation (1.1), f satisfies the condition (1.3), then (1.1) has at least one T-periodic solution.

PROOF. The condition (1.3) implies that $|f|=0$.

\section{REFERENCES}

1. CRONIN, J., "Fixed points and topologica1 degree in nonlinear analysis", Mathematical Surveys, No. 11, American Mathematical Society, Providence, 1964.

2. FENNELL, R.E., Periodic solutions of functional differential equations, J. Math. Ana1. App1. 39 (1972), 198-201.

3. SCHAUDER, J., Der Fixpunktsatz in Funktionalraumen, Studia Math. $\underline{2}$ (1930), 171-180. 


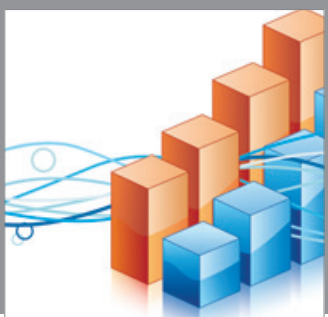

Advances in

Operations Research

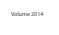

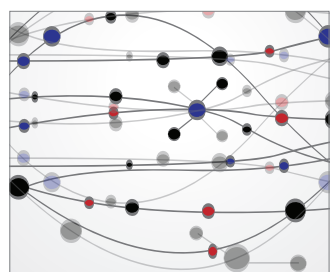

\section{The Scientific} World Journal
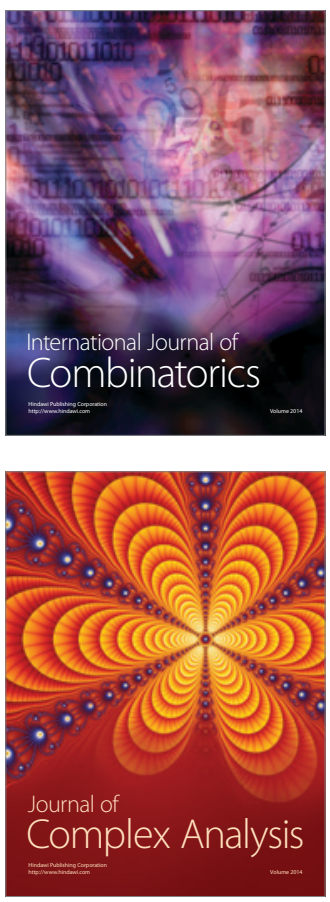

International Journal of

Mathematics and

Mathematical

Sciences
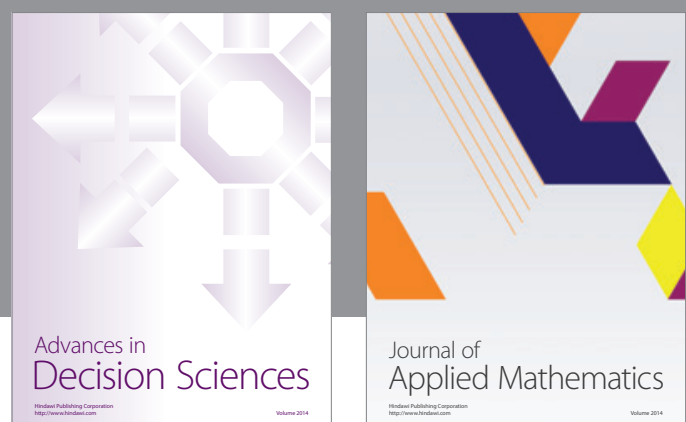

Journal of

Applied Mathematics
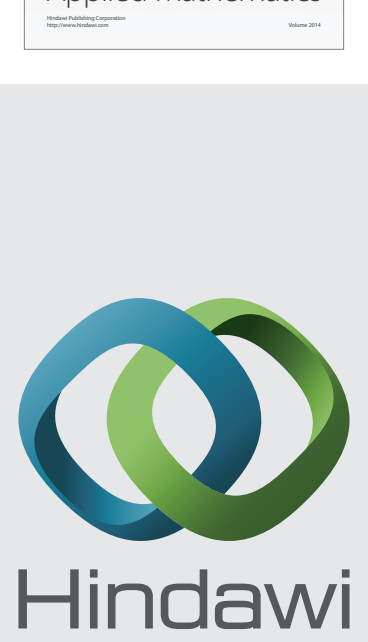

Submit your manuscripts at http://www.hindawi.com
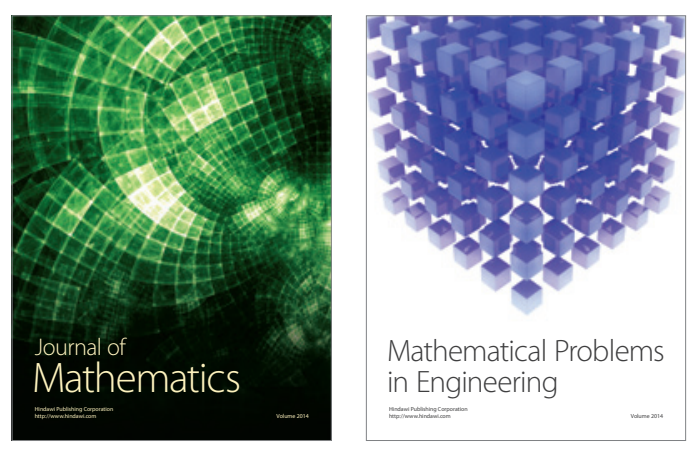

Mathematical Problems in Engineering
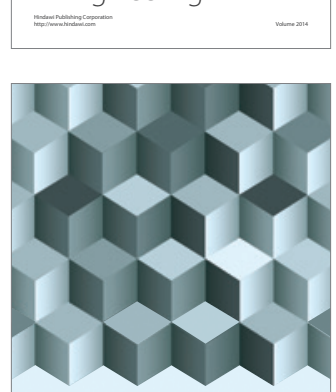

Journal of

Function Spaces
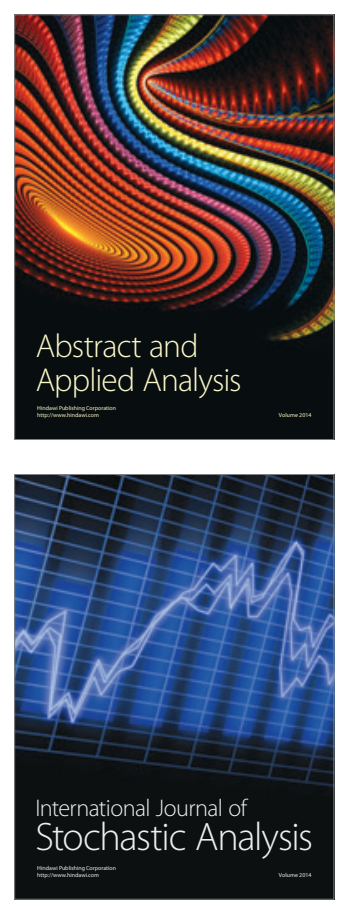

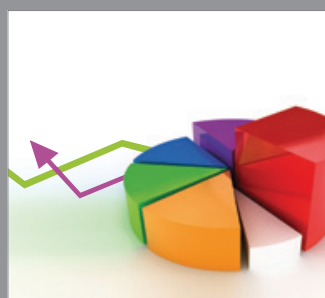

ournal of

Probability and Statistics

Promensencen
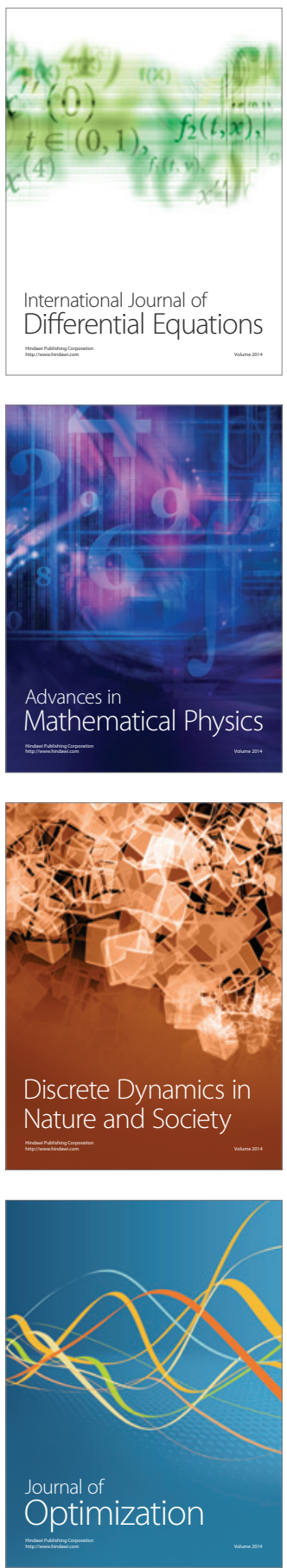